\title{
The value of service reliability
}

\author{
Vincent Benezech $^{\text {a }} \quad$ Nicolas Coulombel ${ }^{\text {a,* }}$ \\ a Université Paris Est, Laboratoire Ville Mobilité Transport, UMR T9403, Marne-la-Vallée, France. \\ * Corresponding author. Address: École des Ponts ParisTech (UMR LVMT), 6-8 Avenue Blaise Pascal, \\ Champs sur Marne, BP 357, F-77455 Marne la Vallée Cedex 2, France. \\ Phone : + 33164152130 ; Fax : +33164152140 ; E-mail : nicolas.coulombel@enpc.fr
}

\begin{abstract}
This paper studies the impact of service frequency and reliability on the choice of departure time and the travel cost of transit users. When the user has $(\alpha, \beta, \gamma)$ scheduling preferences, we show that the optimal head start decreases with service reliability, as expected. It does not necessarily decrease with service frequency, however. We derive the value of service headway $(\mathrm{VoSH})$ and the value of service reliability (VoSR), which measure the marginal effect on the expected travel cost of a change in the mean and in the standard deviation of headways, respectively. The VoSH and the VoSR complete the value of time and the value of reliability for the economic appraisal of public transit projects by capturing the specific link between headways, waiting times, and congestion.
\end{abstract} An empirical illustration is provided, which considers two mass transit lines located in the Paris area.

Key words: Public transportation; Reliability; Headway; Scheduling; Welfare.

\section{Introduction}

The unreliability of transportation systems, in the sense that these systems cannot guarantee perfectly predictable travel times, has various consequences on travelers. It may induce anxiety, cause one to miss a connection (in the case of public transport), or constitute a hindrance to the planning of activities. But the main impact is generally the potential delay at destination(STRATEC and RAND Europe, 2004). Travelers can cope with travel time variability through various means: they can adjust their departure time (Coulombel and de Palma, 2012), change route (Abdel-Aty et al., 1995; Liu et al., 2004) or mode (Chorus et al., 2006), travel somewhere else, or they can decide to report or even to cancel their trip. The preferred strategy is usually to leave with a safety margin (Bates et al., 2001; Li et al., 2010), be it because other alternatives may not be available. This is especially true for transit users, who often have less alternative routes at their disposal than car users to reach their destination.

Following this train of thought, several theoretical works, in line with the seminal contributions of Gaver (1968) and Knight (1974), study the impact of travel time variability on the choice of departure time and the cost of travel (Bates et al., 2001; Coulombel and de Palma, 2013; Fosgerau and Karlström, 2010; Noland and Small, 1995; Siu and Lo, 2009) They adapt the scheduling model popularized by Small (1982) to the context of uncertain travel times. They derive the expected travel cost and the value 
of reliability (VoR), the latter being usually defined as the derivative of the expected travel cost with respect to the standard deviation of travel times. Most works focus on car-users, and although some authors adapt their model to some extent to consider transit users (usually by making departure time a discrete variable), they still fail to take several aspects specific to public transport systems into account. First, they do not distinguish between waiting time and in-vehicle time, which most transit users value differently (Wardman, 2004). Congestion and waiting times are strongly related to headways (and their variability), which is also not modeled in these works. Last, the VoR has two significant drawbacks when applied to public transit: total travel time variability, to which the VoR relates, is hard to measure, but also inconvenient to use for the economic appraisal of public transport projects because the transit operator does not have a direct control over this variable (unlike headway variability or in-vehicle time variability, for instance).

This paper intends to address these issues by adapting the standard scheduling model to the case of public transport. The scope is limited to headway-based services (as opposed to schedule-based ones), for which frequency is high. We study the impact of service headway and reliability on the choice of departure time and the travel cost. Two indicators capture the effect of changes in service characteristics on the expected trip cost: the value of service headway $(\mathrm{VoSH})$ measures the effect of a change in the mean headway, and the value of service reliability (VoSR) that of a change in the standard deviation of headways. The VoSH and VoSR complete the value of time and the VoR in the case of public transit. The former couple relates to headways and their impact on waiting times and in-vehicle congestion, while the latter couple, which originally related to the total travel time in the context of car users, more naturally relates to in-vehicle time for transit users.

The layout of this paper is as follows. Sections 2 and 3 review the theoretical framework, then the main results of renewal theory regarding the link between transit service headways, user-perceived headways, and waiting times. Section 4 presents the model and several findings in the general case. Section 5 elaborates on the case of exponentially distributed headways then provides an empirical illustration, which considers two heavy rail lines located in the Paris area. Section 6 extends the model by introducing in-vehicle congestion, and Section 7 concludes.

\section{Theoretical framework}

There are currently three main modeling frameworks which address the value of travel time variability (Carrion and Levinson, 2012; Li et al., 2010): the mean-variance model, the mean lateness model, and the scheduling model. The first two are based on a descriptive approach: they assume that individuals distaste travel time variability, but do not purport to explain why. These modeling frameworks intend to provide the most efficient specification to estimate the value of reliability.

Scheduling models, on the other hand, provide a micro-economic foundation to the value of reliability. They represent the choice of departure time when individuals face time constraints (e.g. work start time). A first strand of the literature has focused on departure strategies when travel times are uncertain. Gaver (1968) and Knight (1974), who developed the notions of "head start" and "safety margin", respectively, represent two pioneering contributions in this regard. In parallel, another strand of the literature has aimed to model and estimate scheduling preferences when travel times are certain. Building on the works of Gaver (1968) and Vickrey (1969), Small (1982) specified and estimated a scheduling model which has later been widely used in theoretical works. The model is based on the assumption that the traveler's $\operatorname{cost} C$ is a linear function of travel time and schedule delay costs:

$$
C(t)=\alpha T+\beta\left(t^{*}-t-T\right)^{+}+\gamma\left(t+T-t^{*}\right)^{+}+\delta \mathbf{1}\left(t+T-t^{*}\right)
$$


where $(x)^{+}=x$ if $x$ is positive, 0 otherwise, and $\mathbf{1}(x)$ is the Heaviside step function (equal to 1 if $x$ is positive, 0 otherwise). $C(t)$ is the travel disutility when leaving at time $t, T$ the travel time, and $t^{*}-t-T$ the schedule delay. The schedule delay is said to be early if it is positive, late if it is negative. It is measured relatively to a preferred arrival time $t^{*}$, which usually represents the work starting time. The cost of one minute of travel time is $\alpha$; the cost of being one minute early at one's destination is $\beta$ and the cost of being one minute late is $\gamma$. Being late also entails a fixed penalty equal to $\delta$. These parameters are positive (as $C$ is a disutility function); they set the terms of the trade-off between travel time and schedule delay when choosing the departure time. We will refer to (1) as $(\alpha, \beta, \gamma, \delta)$ preferences, or more simply $(\alpha, \beta, \gamma)$ when the late dummy is not included $(\delta=0)$.

In line with Gaver (1968) and Polak (1987), Noland and Small (1995) combine the two above approaches and study the influence of travel time variability on the choice of departure time and the cost of travel under $(\alpha, \beta, \gamma, \delta)$ preferences. Travel time is the sum of a deterministic component and of a random delay, the distribution of which does not vary with the departure time. A key result is the derivation of the minimum expected trip cost when the delay follows a uniform or exponential law. While it is not done in their work, one can use their results to derive the value of reliability (VoR), usually defined as follows (Carrion and Levinson, 2012):

$$
\operatorname{VoR}=\frac{\partial C / \partial \sigma}{\partial C / \partial m}
$$

where $m$ is the trip monetary cost and $\sigma$ the standard deviation of travel times. For $(\alpha, \beta, \gamma, \delta)$ preferences, the parameters are usually expressed in monetary terms and the VoR is simply $\partial C / \partial \sigma$. Fosgerau and Karlström (2010) generalize Noland and Small's work by formally deriving the VoR under less strict assumptions regarding the distribution of the delay. They consider $(\alpha, \beta, \gamma)$ preferences instead of $(\alpha, \beta, \gamma, \delta)$, and assume that the expected travel time is constant. Under these assumptions, the VoR is:

$$
\mathrm{VoR}=(\beta+\gamma) \int_{\frac{\gamma}{\beta+\gamma}}^{1} \Phi^{-1}(s) d s
$$

where $\Phi$ is the cumulative distribution function of the standardized travel time.

Most theoretical works on the VoR focus on car users (e.g. Coulombel and de Palma, 2013; Fosgerau and Karlström, 2010; Noland and Small, 1995). In the case of transit riders, one cannot use the exact same analytical framework for at least two reasons. First, transit services do not run continuously. When choosing their departure time, individuals usually consider the schedule or the frequency of the transit lines that they plan to use (Bowman and Turnquist, 1981; Furth and Muller, 2006). Schedule or headways should thus be explicitly modeled. This point is especially salient as most studies find that individuals have a higher value of waiting time than of in-vehicle time (e.g. Algers et al., 1975; Beesley, 1965; Wardman, 2004). Second, congestion in public transportation is linked to the service headway. It can strongly vary between two consecutive vehicles when headways are irregular (Chen and Liu, 2011). This differs from road congestion which is a more continuous phenomenon, traffic incidents put aside.

Bates et al. (2001) study the choice of departure time and the cost of unreliability in the case of transit users. Their analysis focuses on scheduled services, which leads them to model departure time as 
a discrete variable. ${ }^{1}$ The same choice is operated in Batley (2007), Fosgerau and Karlström (2010), and Fosgerau and Engelson (2011), among others. The underlying assumption is that headways are perfectly reliable, and that the variability of travel time entirely derives from in-vehicle time variability. ${ }^{2}$ This assumption is strong, especially if one considers mass transit lines with high levels of ridership, for which headway regularity is often a significant issue (subsection 5.2 providing an illustrative example). Moreover, these works consider neither the distinction between waiting time and in-vehicle travel time, nor the issue of in-vehicle congestion.

The main purpose of this paper is to study the influence of service reliability (limited here to the dimension of headway regularity ${ }^{3}$ ) on the choice of departure time and the cost of travel. In particular, we show that service reliability impacts the generalized travel cost through three channels: waiting time, schedule delay, and congestion.

\section{Route headways, user-perceived headways and waiting times}

This section reviews the main results of renewal theory regarding the link between the headways of a transit route, the headways perceived by users, and waiting times. We consider first the general case, then the case when the standardized distribution of headways follows a centered exponential law. The reader can refer to Osuna and Newell (1972) or Kleinrock (1975, p. 169) for a proof of these results.

\subsection{General case}

Consider a direct transit line connecting two points $A$ and $B$, which we will refer to as a railway line with no loss of generality. Headways at $A$ are given by a sequence of positive random variables $\left(H_{i}\right)_{i \in \mathbb{Z}}$. They are identically and independently distributed, with probability distribution function (p.d.f.) $\varphi_{H}$ and cumulative distribution function (c.d.f.) $\Phi_{H}$. Headways being positive, $\varphi_{H}$ and $\Phi_{H}$ are both null on $\mathbb{R}^{-*}$. We will assume throughout the text that the distribution of headways has finite moments of all orders. We denote $\mu_{H}$ and $\sigma_{H}$ the mean and the standard deviation of headways; they provide inverse measures of service frequency and reliability, respectively. ${ }^{4}$

A traveler arrives at the train platform in $A$ at time $t$. Given the assumptions, the user-perceived headway, which is defined as the headway that the traveler experiences when arriving at time $t$, is a random variable $H_{U}$ with the following p.d.f.:

$$
\varphi_{U}(x)=\frac{x \varphi_{H}(x)}{\mu_{H}}
$$

The distribution of headways perceived by users differs from the objective distribution. Indeed, when a traveler arrives on the platform in a "random manner" (meaning that he has no dynamic information regarding headways), the longer the headway, the more likely it is for that traveler to arrive in the corresponding time interval. ${ }^{5}$

\footnotetext{
${ }^{1}$ Bates et al. (2001, pp.208-210) treat departure time as a continuous variable when considering the special case where the transit service departure time is random. However, they only provide a very general discussion of this case and do not give any significant result.

${ }^{2}$ Again, Bates et al. (2001) is to the best of our knowledge one the few works to consider headway variability, in a brief manner to boot. Some other works do also consider headway variability (e.g. Bowman and Turnquist, 1981; Furth and Muller, 2006), but they do not use scheduling preferences and resort to ad-hoc cost functions instead.

${ }^{3}$ Service reliability encompasses two major dimensions in the case of headway-based services: headway regularity and in-vehicle time variability. This paper focuses on the former issue.

${ }^{4}$ The mean headway $\mu_{H}$ is inversely commensurate to service frequency, so one should understand "an increase in service frequency" as a decrease in $\mu_{H}$. Similarly, an increase in service reliability corresponds to a decrease in $\sigma_{H}$.

${ }^{5}$ To illustrate this point, consider a transit line with headways of 59.9 and 0.1 minutes with equal probabilities (which is a Bernoulli process with mean 30 minutes). When a traveler arrives at the platform in a random manner,
} 
The waiting time is a stochastic variable $T_{w}$ with p.d.f.:

$$
\varphi_{w}(x)=\frac{1-\Phi_{H}(x)}{\mu_{H}} \text { for } x \in \mathrm{R}^{+}, 0 \text { otherwise }
$$

The mean and the standard deviation of waiting times are (using integration by parts):

$$
\left\{\begin{array}{l}
\mu_{w}=\frac{\mu_{H}}{2}\left(1+\frac{\sigma_{H}^{2}}{\mu_{H}^{2}}\right) \\
\sigma_{w}=\sqrt{\frac{\mu_{H}^{2}}{12}+\frac{\sigma_{H}^{2}}{2}+\frac{\eta_{H} \sigma_{H}^{3}}{3 \mu_{H}}-\frac{\sigma_{H}{ }^{4}}{4 \mu_{H}{ }^{2}}}
\end{array}\right.
$$

where $\eta_{H}$ is the skewness of $\left(H_{i}\right)_{i \in \mathbb{Z}}$. These results call for two comments. First, a change in service reliability (i.e. in $\sigma_{H}$ ) impacts the mean waiting time, even when service headway remains constant. Second, (5) linking the p.d.f. of $H_{i}$ and $T_{w}$, these two variables cannot be standardized simultaneously. Considering our focus on service reliability, we choose to standardize the $\left(H_{i}\right)_{i \in \mathbb{Z}}:^{6}$

$$
H_{i}=\mu_{H}+\sigma_{H} h
$$

where $h$ is a random variable with mean 0 and variance 1 . For reminder, the following relationships link the p.d.f. and c.d.f of $h$ and $H$ :

$$
\varphi_{H}(x)=\frac{\varphi_{h}\left(\frac{x-\mu_{H}}{\sigma_{H}}\right)}{\sigma_{H}} \text { and } \Phi_{H}(x)=\Phi_{h}\left(\frac{x-\mu_{H}}{\sigma_{H}}\right)
$$

\subsection{Exponentially distributed headways}

We will give special attention to the case of a centered exponential distribution. ${ }^{7}$ It leads to closed form solutions and fits the data well to boot (see section 5).

When $h$ follows a centered exponential distribution, we have:

$$
\left\{\begin{array}{l}
\varphi_{h}(x)=e^{-(x+1)} \\
\Phi_{h}(x)=1-e^{-(x+1)}
\end{array} \quad \text { for } x \geq-1\right.
$$

In the exponential case, the condition that headways must be positive is, according to (7), equivalent to constraining $\sigma_{H} \leq \mu_{H}$. Service unreliability as measured by $\sigma_{H}$ cannot exceed $\mu_{H}$ for the standardization of headways to be consistent, the case $\sigma_{H}=\mu_{H}$ corresponding to the standard exponential distribution.

The distribution of headways perceived by users is:

he has practically zero chance of arriving between two trains separated by 0.1 minute. He experiences a mean headway of 59.9 minutes (approximately), which is twice the mean objective headway.

${ }^{6}$ This choice is also empirically supported (see subsection 5.2).

${ }^{7}$ For the sake of brevity, we will often omit the term "centered". 


$$
\left\{\begin{array}{l}
\varphi_{U}(x)=\frac{x}{\mu_{H} \sigma_{H}} e^{-\left(\frac{x+\sigma_{H}-\mu_{H}}{\sigma_{H}}\right)} \\
\Phi_{U}(x)=1-\frac{x+\sigma_{H}}{\mu_{H}} e^{-\left(\frac{x+\sigma_{H}-\mu_{H}}{\sigma_{H}}\right)} \text { for } x \geq \mu_{H}-\sigma_{H}
\end{array}\right.
$$

Lastly, the p.d.f. of waiting times is:

$$
\begin{cases}\varphi_{w}(x)=\frac{1}{\mu_{H}} & \text { for } 0 \leq x \leq \mu_{H}-\sigma_{H} \\ \varphi_{w}(x)=\frac{1}{\mu_{H}} e^{-\left(\frac{x+\sigma_{H}-\mu_{H}}{\sigma_{H}}\right)} & \text { for } x \geq \mu_{H}-\sigma_{H}\end{cases}
$$

Figure 1 illustrates $\varphi_{w}$ for various values of $\sigma_{H}$. When $\sigma_{H}$ tends toward $0, \varphi_{w}$ converges pointwise to the uniform distribution, which corresponds to the case of perfect headway regularity.

FIGURE 1 HERE

Figure 1: Probability distribution function of waiting times (exponential case, $\mu_{H}=1$ )

\section{A scheduling model for headway-based transit services}

\subsection{Model set-up}

An individual wishes to go from point $A$ to point $B$. As previously, a direct transit line connects the two points. The service is headway-based: there is no schedule, or alternatively service frequency is high enough for the user to ignore the schedule and only consider headways when planning his trip. The service is not perfectly reliable, headway variability being modeled by (7). The transit line operator sets the levels of service frequency and reliability, in other words $\mu_{H}$ and $\sigma_{H}$, which are taken as exogenous.

As is common in the literature, we assume that the individual has $(\alpha, \beta, \gamma)$ preferences. The relevancy of $(\alpha, \beta, \gamma)$ preferences for modeling the departure time choice is actually controversial. While Noland et al. (1998) find that $(\alpha, \beta, \gamma)$ preferences well fit the behavior of individuals in their survey, Tseng and Verhoef (2008) raise the objection that the assumption of time-invariant shadow prices is unrealistic, a point corroborated by their empirical analysis. In addition, Börjesson et al. (2012) find that $(\alpha, \beta, \gamma)$ preferences do not well capture the aversion of individuals to travel time variability. While acknowledging these limitations, $(\alpha, \beta, \gamma)$ preferences are widely used in this research field, simple to handle from a mathematical standpoint, and yield closed-form solutions in our application in section 5. These various points led us to keep this functional form. ${ }^{8}$

The individual has standard $(\alpha, \beta, \gamma)$ preferences except that he values in-vehicle time and waiting time differently. The generalized cost of travel (or travel disutility) is:

$$
C(t)=\alpha_{w} T_{w}+\alpha_{v} T_{v}+\beta\left(t *-t-T_{w}-T_{v}\right)^{+}+\gamma\left(t+T_{w}+T_{v}-t^{*}\right)^{+}
$$

\footnotetext{
${ }^{8}$ We could easily apply the same framework to another utility function. Closed-form solutions would not be guaranteed in the exponential case, however.
} 
where $T_{w}$ is the platform waiting time and $T_{v}$ the in-vehicle time. The real parameters $\left(\alpha_{w}, \alpha_{v}, \beta, \gamma\right)$ are positive. A common result of the empirical literature on the value of time is $\alpha_{w}>\alpha_{v}$ (Wardman, 2004): waiting yields less utility than being in a vehicle. The model remains valid when $\alpha_{w} \leq \alpha_{v}$. Most empirical studies on scheduling preferences also find that $(\alpha, \beta, \gamma)$ are ordered as follows: $\beta<\alpha<\gamma$ (Bates et al., 2001; Carrion and Levinson, 2012). Added to the fact $\beta>\alpha$ that leads to counterintuitive behaviors (such as preferring to wait on the platform or to spend more time in the vehicle rather than to get early at one's destination), we assume: $0<\beta<\alpha=\min \left(\alpha_{w}, \alpha_{v}\right)$ and $\gamma>0$.

Waiting time $T_{w}$ is a random variable, its distribution being given by (5). Onboard travel time $T_{v}$ is on the other hand deterministic and constant. This assumption is made for the sake of simplicity, as computations are quite complex and hard to follow when both in-vehicle times and waiting times vary. Our results can easily be extended to the case with variable in-vehicle time, as long as $T_{v}$ and $T_{w}$ are not correlated. $^{9}$

Waiting time variability has a twofold source in our model, as brought to light by (5) and (6). First, the user only knows the headway distribution of the railway line (i.e. $\varphi_{h}$ ), not the exact schedule. Even in the case of perfectly regular headways, he will still arrive at a random time between two trains. Second, headways are not perfectly reliable, which increases further waiting time variability. Indeed, (5) shows that waiting times follow a distribution which is uniform when headway reliability is perfect $\left(\sigma_{H}=0\right)$, but progressively changes as reliability declines.

We denote $m=t^{*}-t-T_{v}$ the head start. It is the time allocated to waiting, which can be positive or negative. Travel cost becomes:

$$
C(m)=\alpha_{w} T_{w}+\alpha_{v} T_{v}+\beta\left(m-T_{w}\right)^{+}+\gamma\left(T_{w}-m\right)^{+}
$$

If $T_{w}<m$, the traveler arrives early at his destination, if $T_{w}>m$ he is late. Because $T_{w}$ is positive, choosing a strictly negative head start implies arriving late with certainty.

\subsection{Optimal head start}

The traveler chooses the head start which minimizes his expected travel disutility:

$$
\bar{C}(m)=\alpha_{w} \mu_{w}+\alpha_{v} T_{v}+\beta \int_{0}^{m}(m-x) \varphi_{w}(x) d x+\gamma \int_{m}^{+\infty}(x-m) \varphi_{w}(x) d x
$$

The first order condition is $\beta \Phi_{w}\left(m^{*}\right)-\gamma\left(1-\Phi_{w}\left(m^{*}\right)\right)=0$. The optimal head start is:

$$
m^{*}=\Phi_{w}^{-1}(\kappa)
$$

where $\kappa=\gamma / \beta+\gamma$ is the relative shadow price of being late.

We find an equation identical to Fosgerau and Karlström (2010) except that it involves the c.d.f. of waiting time instead of travel time. In our set-up, the choice of the head start $m$ only impacts the expected scheduling cost within the total expected cost of travel. It bears no influence on the travel time cost component $\alpha_{w} \mu_{w}+\alpha_{v} T_{v}$, which is independent of $m$. This explains why $m^{*}$ only depends on the

\footnotetext{
${ }^{9}$ There is likely some linkage between the variability of headways and in-vehicle times, and thus of waiting times and in-vehicle times. However, there is a direct link between headway variability and waiting time variability, which is less clear for headway variability and in-vehicle time variability. Were the connection between the two to be established (literature on this topic being currently seldom to the best of our knowledge), we could model $T_{v}$ as a random variable function of $H$ and take this phenomenon into account.
} 
scheduling parameters $\beta$ and $\gamma$ and not on the values of time $\alpha_{w}$ and $\alpha_{v}$. The safety margin increases with $\gamma$ and decreases with $\beta$. The more important it is to be on time, the more extra time one plans for his trip. When $\gamma$ tends towards $+\infty$, the optimal margin tends towards $\Phi_{w}{ }^{-1}(1)$, the maximum waiting time, also equal to the maximum headway. Individuals who must be on time at all costs consider the worst case scenario and take the necessary head start.

When service reliability is perfect $\left(\sigma_{H}=0\right)$, the optimal safety margin is $m_{d e t}^{*}=\kappa \mu_{H}$. It is equal to the average waiting time $\mu_{H} / 2$ when $\beta=\gamma$. If $\beta<\gamma$, the individual dislikes being late more than being early and plans more time for waiting than the mean waiting time, and vice versa.

The effects of marginal changes in service headway and reliability on the optimal head start are given by (see Appendix):

$$
\left\{\begin{array}{l}
\frac{d m^{*}}{d \mu_{H}}=\frac{\kappa-\Phi_{H}\left(m^{*}\right)}{1-\Phi_{H}\left(m^{*}\right)} \\
\frac{d m^{*}}{d \sigma_{H}}=\frac{1}{\sigma_{H}}\left(m^{*}+\frac{\Phi_{H}\left(m^{*}\right)-\kappa}{1-\Phi_{H}\left(m^{*}\right)} \mu_{H}\right)
\end{array}\right.
$$

The shape of the headway distribution largely determines the impact of $\mu_{H}$ and of $\sigma_{H}$ on the optimal head start. Nevertheless, we show three main results in the general case (see Appendix for proof). First, the optimal head start $m^{*}$ is always greater than $m^{*}{ }_{d e t}$. As expected, headway variability causes individuals to take a bigger margin than when headways are perfectly regular. Second, $d m^{*} / d \mu_{H}<1$. When the operator increases the service headway (while keeping reliability at the same level), the user increases his margin by less than the increase in $\mu_{H}$. Lastly, $d m^{*} / d \sigma_{H}>0$ : the less reliable the service, the greater the head start. This is again coherent with intuition.

The sign of $d m^{*} / d \mu_{H}$ is not clear cut in the general case, however. While one would expect $d m^{*} / d \mu_{H}>0$, which indeed is usually the case, the opposite can occur for fat-tailed distributions. An increase in the service headway causes then travelers to reduce their head start. This counterintuitive result stems from the disjunction between the objective and subjective distributions of headways. ${ }^{10}$

\subsection{Values of service headway and reliability}

Using the first order condition, we obtain the minimum expected travel cost:

$$
C^{*}=\bar{C}\left(m^{*}\right)=\alpha_{v} T_{v}+\left(\alpha_{w}-\beta\right) \mu_{w}+(\beta+\gamma) \int_{m^{*}}^{+\infty} x \varphi_{w}(x) d x
$$

or equivalently:

$$
C^{*}=\alpha_{v} T_{v}+\left(\alpha_{w}+\gamma\right) \mu_{w}-(\beta+\gamma) \int_{0}^{m^{*}} x \varphi_{w}(x) d x
$$

When headways are perfectly reliable, the travel cost is:

\footnotetext{
${ }^{10}$ In the example discussed in footnote 5 , slightly increasing $\mu_{H}$ would actually be beneficial to transit users as they would have more chance to board the trains with very short headways, hence a shorter safety margin.
} 


$$
C_{d e t}^{*}=\alpha_{v} T_{v}+\alpha_{w} \frac{\mu_{H}}{2}+\beta \kappa \frac{\mu_{H}}{2}
$$

The first two terms are the time cost. The last term is the scheduling cost; unlike in the standard model (e.g. Fosgerau and Karsltröm, 2010), it is not null because waiting times exhibit variability even when headways are perfectly reliable. ${ }^{11}$

To study the impact of service characteristics on the travel cost, we introduce two indicators. The value of service headway (VoSH) and the value of service reliability (VoSR) are the derivatives of the minimum expected travel cost with respect to the mean and the standard deviation of headways, respectively: ${ }^{12}$

$$
\left\{\begin{array}{l}
\operatorname{VoSH}=\frac{\partial C^{*}}{\partial \mu_{H}} \\
\operatorname{VoSR}=\frac{\partial C^{*}}{\partial \sigma_{H}}
\end{array}\right.
$$

The VoSH and the VoSR are closely related to the VoT and the VoR. Actually, the model of Fosgerau and Karlström (2010) can easily be adapted to derive the value of waiting time (VoWT) and the value of waiting time variability (VoWR). ${ }^{13}$ The couple (VoWT, VoWR) would be formally equivalent to the couple (VoSH, VoSR), except that it would use the waiting time metrics instead of the headway metrics. For headway-based transit services, the headway metrics is the most relevant one, however. Headways are easier to measure than waiting times. Transit operators control headways, not waiting times. ${ }^{14}$ And congestion is easier to introduce with headways than with waiting times (see section 6).

Differentiating (18) yields the VoSH and the VoSR:

$$
\left\{\begin{array}{l}
\operatorname{VoSH}=\frac{\alpha_{w}+\gamma}{2}\left(1-\frac{\sigma_{H}^{2}}{\mu_{H}^{2}}\right)+\frac{\beta+\gamma}{\mu_{H}} \int_{0}^{m^{*}}\left(\Phi_{H}(x)-\Phi_{w}(x)\right) d x \\
\operatorname{VoSR}=\left(\alpha_{w}+\gamma\right) \frac{\sigma_{H}}{\mu_{H}}+\gamma \frac{\mu_{H}-m^{*}}{\sigma_{H}}-(\beta+\gamma) \frac{m^{*}}{\sigma_{H}}+2 \frac{\beta+\gamma}{\sigma_{H}} \int_{0}^{m^{*}} \Phi_{w}(x) d x
\end{array}\right.
$$

In the general case, the relationship between the VoSH and the various model parameters is complex. Similarly, comparative statics for the VoSR are not straightforward. We turn to the exponential case: this allows us to analytically derive the VoSH and the VoSR while providing a good approximation of real-case data, as we will see for two mass transit lines of the Paris area.

\section{Application: the exponential case}

From this point on, we assume that headways follow a centered exponential distribution:

\footnotetext{
${ }^{11}$ Interestingly, the term $\beta \mu_{H} / 2$ is similar to the term $\beta \kappa N / s$ that appears in the equilibrium cost of the bottleneck model of road congestion.

${ }^{12}$ We thereby extend the work of Fosgerau (2009) by completing his analysis on the value of headway in the case of deterministic headways to the case of variable headways.

${ }^{13}$ Indeed, one can apply the model of Fosgerau and Karlström to the case where the travel time is the sum of a constant in-vehicle time and a variable waiting time. Travel time variability would entirely stem from waiting time variability, and the various results would still apply using the waiting time distribution instead of the travel time distribution.

${ }^{14}$ To be precise, operators can control waiting times, but indirectly (through headways) and not perfectly. For instance, even if headway regularity is perfect, waiting times remain variable. It is also likely that users have a good knowledge of the frequency of the lines they use but not of the distribution of waiting times.
} 


$$
H_{i}=\mu_{H}+\sigma_{H} h
$$

where $h$ is a random variable with centered exponential law (which has -1 as a minimum). As underlined in subsection 3.1, the condition that headways must be positive implies that this specification only makes sense for $\sigma_{H} \leq \mu_{H}$.

\subsection{Analytical derivation}

Two regimes arise in the exponential case (see Appendix and Figure 1 for an intuitive interpretation). In the first mode, headway variability is low (in relative terms): $\sigma_{H} / \mu_{H} \leq 1-\kappa$. The optimal head start and the minimum cost are:

$$
\left\{\begin{array}{l}
m^{*}=\kappa \mu_{H}=m_{d e t}^{*} \\
C^{*}=\alpha_{v} T_{v}+\left(\alpha_{w}+\gamma\right) \mu_{w}-\gamma \kappa \frac{\mu_{H}}{2}=C_{d e t}^{*}+\left(\alpha_{w}+\gamma\right) \frac{\sigma_{H}{ }^{2}}{2 \mu_{H}}
\end{array}\right.
$$

In this regime, service reliability is good enough so that individuals do not change their departure time compared to the deterministic case. The expected travel cost does increase, however, as a result of two concurring phenomena. Headway variability increases the mean waiting time, which is captured by the term $\alpha_{w} \sigma_{H}{ }^{2} /\left(2 \mu_{H}\right)$. It also leads to greater waiting time variability, hence a greater scheduling cost. The term $\gamma \sigma_{H}{ }^{2} /\left(2 \mu_{H}\right)$ reflects this second mechanism.

When service reliability is poor $\left(\sigma_{H} / \mu_{H} \geq 1-\kappa\right)$, the system tips over the exponential regime. The optimal head start and the expected trip cost are:

$$
\left\{\begin{array}{l}
m^{*}=\mu_{H}-\sigma_{H}+\sigma_{H} \ln \left(\frac{1}{1-\kappa} \frac{\sigma_{H}}{\mu_{H}}\right) \\
C^{*}=\alpha_{v} T_{v}+\alpha_{w} \mu_{W}+\beta\left(\frac{\mu_{H}{ }^{2}-\sigma_{H}^{2}}{2 \mu_{H}}+\sigma_{H} \ln \left(\frac{1}{1-\kappa} \frac{\sigma_{H}}{\mu_{H}}\right)\right)
\end{array}\right.
$$

Individuals respond to the poorer service reliability by adjusting their safety margin. As expected, the margin increases with the service headway and the service unreliability: $d m^{*} / d \mu_{H}=1-\sigma_{H} / \mu_{H}>0$ and $d m^{*} / d \sigma_{H}=\ln \left(\sigma_{H} / \mu_{H} \cdot(\beta+\gamma) / \beta\right)>0$.

Figure 2 compares the optimal head start in the deterministic case and for three values of $\sigma_{H}$. In the former case, the optimal head start increases linearly in $\kappa$ (measuring the relative cost of being late). For low values of $\kappa$, the head start does not depend on $\sigma_{H}$. When $\kappa$ exceeds a certain threshold (itself decreasing with $\sigma_{H}$ ), individuals shift to the exponential regime. As $\kappa$ increases, they increase more and more their margin to insure themselves against the risk of being late caused by service unreliability. When $\kappa$ tends toward 1, the head start tends toward $+\infty$. Since headways are unbounded for exponential distributions, insuring oneself against the worst case scenario implies taking an infinite safety margin.

FIGURE 2 HERE

Figure 2: Influence of headway variability on the head start (exponential case, $\mu_{H}=1$ )

We can use (23) and (24) to derive the value of service headway (VoSH) for the two regimes: 


$$
\begin{cases}\operatorname{VoSH}=\left(1-\frac{\sigma_{H}^{2}}{\mu_{H}^{2}}\right) \frac{\alpha_{w}}{2}+\left(1-\kappa-\frac{\sigma_{H}^{2}}{\mu_{H}^{2}}\right) \frac{\gamma}{2} & \text { if } \quad \frac{\sigma_{H}}{\mu_{H}} \leq 1-\kappa \\ \operatorname{VoSH}=\left(1-\frac{\sigma_{H}^{2}}{\mu_{H}^{2}}\right) \frac{\alpha_{w}}{2}+\left(1-\frac{\sigma_{H}}{\mu_{H}}\right)^{2} \frac{\beta}{2} & \text { if } \quad \frac{\sigma_{H}}{\mu_{H}} \geq 1-\kappa\end{cases}
$$

As expected, the VoSH increases with $\alpha_{w}, \beta$, and $\gamma .{ }^{15}$ The more the user values not waiting or being on time, the more he enjoys better transit service frequency. Furthermore, the VoSH decreases with $\sigma_{H} / \mu_{H}$. Improving service frequency is more beneficial when headway variability is low (in relative terms). ${ }^{16}$

Similarly, we can derive the value of service reliability (VoSR):

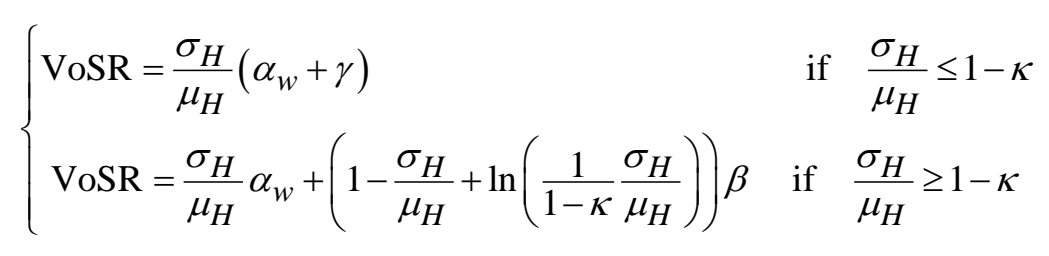

Similarly, the VoSR increases with $\alpha_{w}, \beta$, and $\gamma$. Headway variability increases the mean waiting time, which explains that the higher $\alpha_{w}$, the more a degradation in service reliability would cost. In addition, headway irregularity increases waiting time variability. This increases the scheduling disutility, hence the increase with $\beta$ and $\gamma .{ }^{17}$ Unlike the VoSH, the VoSR increases with $\sigma_{H} / \mu_{H}$ instead of decreasing. Thus, there exists a cut-off level above which it is more beneficial to improve service reliability than service frequency (Figure 3).

FIGURE 3 HERE

Figure 3: Influence of service reliability on the VoSH and the VoSR (exponential case, $\beta=0.8, \gamma=3, \alpha_{w}=2$ )

\subsection{Empirical illustration}

We use data on observed headways during the morning peak hour to estimate the headway distribution of two mass transit lines of the Paris area, the RER A and the RER E. Data were provided by the railway operator, Transilien, in the case of the RER E, and were collected using real-time data available on the web in the case of the RER A. Both databases provide records for a time period of 3 months in 2011. For the RER A, headways are measured at the station named Nation, which is located on the eastern frontier of Paris, and for westbound trains. For the RER E, headways are measured at Bondy, which is outside of Paris, again for westbound trains.

The RER A is one of the most used transit lines in the world, with more than one million riders a day. At the morning peak hour, headways are short but the high congestion level hampers operations,

\footnotetext{
${ }^{15}$ Less intuitive is the fact that, in the exponential regime, the VoSH does not depend on $\gamma$. This property is likely specific to the exponential distribution, but further investigation would be necessary to assert this point.

${ }^{16}$ This point was not trite considering the example in footnote 5 . When headways are highly irregular, increasing the mean headway could lead to a better allocation of travelers between trains, and subsequently to a decrease of the expected cost of travel.

${ }^{17}$ In a way symmetric to what happens for the VoSH, the VoSR does not depend on $\beta$ in the deterministic regime. Again, we did not find an easy interpretation of this intriguing result, which is most surely linked to the specificities of the exponential distribution.
} 
leading to irregular headways. Parametric estimation of the headway distribution yields $\mu_{H}=2.4$ and $\sigma_{H}=0.9$, the exponential distribution fitting the data remarkably well (Figure 4a).

\section{FIGURE 4 HERE}

Figure 4: Density of waiting times during the morning peak hour for westbound passengers arriving randomly at Nation and Bondy stations (Web-sourced data for RER A, Transilien data for RER E)

The RER E is a newer and less central line than the RER A, with about 300,000 travelers per day. Frequency is lower than for the RER A $\left(\mu_{H}=7.8\right)$, for a similar service reliability $\left(\sigma_{H}=1.0\right)$. Again, the exponential distribution fits the data well, although less so than for the RER A (Figure 4b).

To illustrate our findings, we consider a traveler who has the following preference parameters: $\alpha_{v}=1, \alpha_{w}=2, \beta=0.8, \gamma=3{ }^{18}$ His journey on the RER (A or E) is 10 minutes long. Under these assumptions, the VoSH is equal to 1.02 and the VoSR to 1.71 for the RER A. For the RER E, the values are 1.27 and 0.64 , respectively. Judging from the values of the VoSH and the VoSR, it will likely be more beneficial to improve reliability on the RER A and frequency on the RER E. To confirm this point, we simulate in Table 1 the impact of an increase in either frequency or reliability on the expected travel cost, which is broken down into three components, in-vehicle time, waiting time and schedule delay, as apparent in equation (14). As expected, the best scenario is "Reliability improvement" for the RER A and "Frequency improvement" for the RER E . This basic simulation hints that future investments should focus on improving service reliability on the RER A and increasing frequency of the RER E. ${ }^{19}$

Table 1: Simulation of 2 improvement scenarios for the RER A and the RER E

\begin{tabular}{|c|c|c|c|c|c|c|c|c|}
\hline & \multirow[b]{2}{*}{ Scenario } & \multirow[b]{2}{*}{$\mu_{H}$} & \multicolumn{6}{|c|}{ Expected cost } \\
\hline & & & $\sigma_{H}$ & $\begin{array}{l}\text { In-vehicle } \\
\text { time }\end{array}$ & $\begin{array}{l}\text { Waiting } \\
\text { time }\end{array}$ & $\begin{array}{c}\text { Schedule } \\
\text { delay }\end{array}$ & Total & $\begin{array}{l}\text { Relative } \\
\text { decrease }\end{array}$ \\
\hline \multirow{3}{*}{$\begin{array}{c}\text { RER } \\
\text { A }\end{array}$} & Actual & 2.4 & 0.9 & 10 & 2.74 & 1.24 & 13.98 & - \\
\hline & $\begin{array}{c}\text { Frequency } \\
\text { improvement }\end{array}$ & 1.6 & 0.9 & 10 & 2.11 & 1.15 & 13.25 & $5,2 \%$ \\
\hline & $\begin{array}{c}\text { Reliability } \\
\text { improvement }\end{array}$ & 2.4 & 0.1 & 10 & 2.40 & 0.76 & 13.17 & $5,8 \%$ \\
\hline \multirow{3}{*}{$\begin{array}{c}\text { RER } \\
\text { E }\end{array}$} & Actual & 7.8 & 1 & 10 & 7.93 & 2.66 & 20.58 & - \\
\hline & $\begin{array}{l}\text { Frequency } \\
\text { improvement }\end{array}$ & 7 & 1 & 10 & 7.14 & 2.42 & 19.57 & $4,9 \%$ \\
\hline & $\begin{array}{c}\text { Reliability } \\
\text { improvement }\end{array}$ & 7.8 & 0.2 & 10 & 7.81 & 2.47 & 20.28 & $1,5 \%$ \\
\hline
\end{tabular}

\footnotetext{
${ }^{18}$ Regarding the triplet $(\alpha, \beta, \gamma)$, Bates et al. (2001) indicate the ratios 1:0.8:3 as typical from the literature. A ratio of 2 for $\alpha_{w} / \alpha_{v}$ is also standard (Wardman, 2004).

${ }^{19}$ Obviously, the investment costs of improving service frequency and reliability should also be factored in the analysis to confirm this point.
} 


\section{Extension: in-vehicle congestion}

Headway has an impact on waiting times, but also on in-vehicle congestion (see section 2). When headways are irregular, longer headways are associated with both longer waiting times and greater passenger vehicle loads, hence a "double punishment" (and vice versa for shorter headways). To take this effect into account, we amend the model by linking the value of in-vehicle time to the number of passengers $N$ :

$$
C(t)=\alpha_{w} T_{w}+\alpha_{v} \Theta(N) T_{v}+\beta\left(t^{*}-t-T_{w}-T_{v}\right)^{+}+\gamma\left(t+T_{w}+T_{v}-t^{*}\right)^{+}
$$

where $\Theta(N)$ represents in-vehicle discomfort and increases with $N$. For the sake of exposition, we choose a simple specification for $\Theta$ :

$$
\begin{cases}\Theta(N)=1 & \text { if } N \leq N_{0} \\ \Theta(N)=k & \text { if } N>N_{0}\end{cases}
$$

If the number of passengers is greater than $N_{0}$, the vehicle is overcrowded. The value of in-vehicle time is penalized by a factor $k>1$. Otherwise, it retains its former value $\alpha_{v}$.

We assume that the rate of new travelers arriving on the platform is constant and equal to $q$. All travelers, our individual included, are impatient: they board the first train regardless of congestion. Furthermore, we consider that trains are empty when they arrive at A, which is the departure station. Under these assumptions, a simple relationship links the passenger vehicle load to the headway: $N=q H$. We rewrite $\Theta$ as a function of $H$ instead of $N$ :

$$
\left\{\begin{array}{cl}
\Theta(H)=1 & \text { if } H \leq H_{0}=N_{0} / q \\
\Theta(H)=k & \text { if } H>H_{0}
\end{array}\right.
$$

Our individual being impatient, introducing congestion does not change the choice of departure time since headways are not time dependent. ${ }^{20}$ It changes the expected travel cost, however (note that we still consider exponential headways):

$$
\begin{cases}C^{*}=\alpha_{v} T_{v} E\left(\Theta\left(H_{U}\right)\right)+\alpha_{w} \mu_{W}+\gamma \frac{\mu_{H}}{2}\left(1-\kappa+\frac{\sigma_{H}^{2}}{\mu_{H}^{2}}\right) & \text { if } \frac{\sigma_{H}}{\mu_{H}} \leq 1-\kappa \\ C^{*}=\alpha_{v} T_{v} E\left(\Theta\left(H_{U}\right)\right)+\alpha_{w} \mu_{W}+\beta\left(\frac{\mu_{H}^{2}-\sigma_{H}^{2}}{2 \mu_{H}}+\sigma_{H} \ln \left(\frac{1}{1-\kappa} \frac{\sigma_{H}}{\mu_{H}}\right)\right) & \text { if } \frac{\sigma_{H}}{\mu_{H}} \geq 1-\kappa\end{cases}
$$

Note that because we take the user point of view, we take the expectation of $\Theta\left(H_{U}\right)$ and not of $\Theta(H)$. Computations give:

\footnotetext{
${ }^{20}$ If our individual is patient, the problem is more complex. If the first train to arrive is crowded, the individual can wait for the next train hoping that it will not be crowded. This possibility modifies the problem of the choice of departure time, and depending on how much the individual dislikes congestion, he might increase his safety margin to insure himself against this eventuality. For the sake of simplicity, we do not consider this possibility. This model does not include inter-personal variability either, in the sense that all passengers experience the same level of congestion while in reality, some passengers are comfortably sitting, even in overcrowded vehicles.
} 


$$
\begin{cases}E\left(\Theta\left(H_{U}\right)\right)=k & \text { if } H_{0} \leq \mu_{H}-\sigma_{H} \\ E\left(\Theta\left(H_{U}\right)\right)=1+(k-1) \frac{H_{0}+\sigma_{H}}{\mu_{H}} e^{-\left(\frac{H_{0}+\sigma_{H}-\mu_{H}}{\sigma_{H}}\right)} & \text { if } H_{0} \geq \mu_{H}-\sigma_{H}\end{cases}
$$

If $H_{0}<\mu_{H}-\sigma_{H}$, all trains are congested. The expected trip cost increases by a constant $(k-1) \alpha_{v} T_{v}$. If $H_{0} \geq \mu_{H}-\sigma_{H}$, only trains with a headway above $H_{0}$ are congested. This model, seemingly simple at first glance, leads to strong discontinuities when $H_{0}$ is close to $\mu_{H}$ (Figure 5).

When $H_{0}$ increases, congestion is less severe, the case with no congestion corresponding to the limit case $H_{0}=+\infty$. For a fixed headway variability $\sigma_{H}$, the travel cost always decreases with $H_{0}$. The reverse is not true: $H_{0}$ being fixed, the expected travel cost does not always increase with $\sigma_{H}$. There are two polar cases. If $H_{0} \geq \mu_{H}$, frequency is high enough and no train is congested when $\sigma_{H}=0$. In this "normal regime", congestion only arises because headway variations can cause headways to exceed $H_{0}$. In the "degraded regime" $\left(H_{0}<\mu_{H}\right)$, all trains are congested when the service is perfectly reliable. The travel cost jumps from $C_{d e t}^{*}$ to $C_{d e t}^{*}+(k-1) \alpha_{v} T_{v}$ when $\sigma_{H}=0$. This time, service unreliability causes some trains to run with headways shorter than $H_{0}$ and be uncongested. This explains why the travel cost may fall when $\sigma_{H}$ increases in the degraded regime (Figure 5).

\section{FIGURE 5 HERE}

Figure 5: Influence of $H_{0}$ on the expected travel cost curve (exponential case, $\alpha_{v}=1, T_{v}=3, \alpha_{w}=2, \beta=0.8, \gamma=3, k=2$ and $\mu_{H}=1$ )

We now derive the VoSH and the VoSR to establish these points more firmly. ${ }^{21}$ If $\sigma_{H}<\mu_{H}-H_{0}$, all trains are congested, and the VoSH and VoSR are the same as before. If $\sigma_{H} \geq \mu_{H}-H_{0}$, the VoSH and the VoSR are modified as follows ( $c$ denoting the model with congestion, and $u$ without):

$$
\left\{\begin{array}{l}
\operatorname{VoSH}_{c}=\operatorname{VoSH}_{u}+(k-1) \alpha_{v} T_{v} \frac{\left(H_{0}+\sigma_{H}\right)\left(\mu_{H}-\sigma_{H}\right)}{\mu_{H}{ }^{2} \sigma_{H}} e^{-\left(\frac{H_{0}+\sigma_{H}-\mu_{H}}{\sigma_{H}}\right)} \\
\operatorname{VoSR}_{c}=\operatorname{VoSR}_{u}+(k-1) \alpha_{v} T_{v} \frac{1}{\mu_{H}}\left(1+\frac{\left(H_{0}+\sigma_{H}\right)\left(H_{0}-\mu_{H}\right)}{\sigma_{H}{ }^{2}}\right) e^{-\left(\frac{H_{0}+\sigma_{H}-\mu_{H}}{\sigma_{H}}\right)}
\end{array}\right.
$$

Congestion increases the $\mathrm{VoSH}: \mathrm{VoSH}_{c} \geq \mathrm{VoSH}_{u}$ seeing that the second term in (32) is always positive. In the degraded regime, the VoSH is discontinuous with a spike at $\sigma_{H}=\mu_{H}-H_{0}$ (Figure 6). This is the limit between the "fully degraded" mode (all trains are congested) and the "partly degraded" mode (only a fraction of them are). From this point on, improving service frequency leads to more and more trains being uncongested, hence the surge in the VoSH.

\footnotetext{
${ }^{21}$ We still call VoSH and VoSR the derivatives of the expected cost with respect to $\mu_{H}$ and $\sigma_{H}$. However, it is worth noting that these are now different from the willingness-to-pay of travelers for an increase in frequency or reliability. Indeed, passengers are not likely to take a diminution of congestion into account when faced with an improvement in service frequency or reliability, because congestion is not intrinsic to passengers' preferences. However, the VoSH or VoSR can still be used to assess the socio-economic consequences of a change in operations.
} 
Contrary to $\mathrm{VoSH}_{c}, \mathrm{VoSR}_{c}$ can be lower than $\mathrm{VoSR}_{u}$ in the degraded regime. In (32), the term $1+\left(H_{0}+\sigma_{H}\right)\left(H_{0}-\mu_{H}\right) / \sigma_{H}^{2}$ is positive in the normal regime $\left(H_{0} \geq \mu_{H}\right)$, but negative in the degraded mode when $\sigma_{H} \in\left[\mu_{H}-H_{0} ; 1 / 2\left(\mu_{H}-H_{0}+\sqrt{\left(\mu_{H}-H_{0}\right)\left(\mu_{H}+3 H_{0}\right)}\right)\right]$. On this interval, increasing headway variability reduces the number of congested trains, which causes the travel cost to fall.

Headway variability entails a "double punishment" in the normal regime. Travel time variability increases, raising the mean schedule delay cost, and some trains get overcrowded. Service unreliability is less detrimental in the degraded mode: it still raises schedule delay costs, but lowers the mean congestion cost by reducing the number of overcrowded trains. These results stem from the properties of the congestion function presented in equation (28): because it is a step function, it does not increase gradually and convexly with the number of passengers. In particular, in the normal regime, it is convex around $\mu_{H}$, but in the degraded regime it is concave around $\mu_{H} \cdot{ }^{22}$ If the congestion function was convex, headway variability would always raise the mean congestion cost, and conversely for a concave function. ${ }^{23}$

\section{FIGURE 6 HERE}

Figure 6: Influence of congestion intensity $H_{0}$ on the VoSH (exponential case, $\alpha_{v}=1, T_{v}=3, \alpha_{w}=2, \beta=0.8, \gamma=3, k=2$ and $\mu_{H}=1$ )

\section{FIGURE 7 HERE}

Figure 7: Influence of congestion intensity $H_{0}$ on the VoSR (exponential case, $\alpha_{v}=1, T_{v}=3, \alpha_{w}=2, \beta=0.8, \gamma=3, k=2$ and $\mu_{H}=1$ )

\section{Conclusion}

Service frequency and reliability impact the travel cost of transit users through three channels: waiting time, schedule delay and congestion. Transit users behave strategically and adjust their departure time in order to minimize these costs. The safety margin, which represents the time allocated to waiting, decreases with service reliability, as expected. It increases with service headway, except for specific distributions of headways.

The values of service headway (VoSH) and service reliability (VoSR) measure the marginal effect on the expected travel cost of a change in the mean and in the standard deviation of headways, respectively. They complete the values of in-vehicle time and in-vehicle time variability by capturing the influence that headways have on waiting times and congestion. Furthermore, they can easily be computed: they relate to the distribution of headways, which are easily measured unlike waiting times or total travel times. This makes the VoSH and the VoSR well suited for the economic appraisal of public transport projects.

In the case of exponentially distributed headways with no congestion, we show that the VoSH is actually not constant and decreases with service reliability. Conversely, the VoSR increases with the

\footnotetext{
${ }^{22}$ To be specific, equation (29) implies that the congestion function satisfies $\mathrm{E}\left(\Theta\left(H_{U}\right)\right) \geq \Theta\left(\mu_{H}\right)$ for $H_{0} \geq \mu_{H}$ (normal regime) and $\mathrm{E}\left(\Theta\left(H_{U}\right)\right) \leq \Theta\left(\mu_{H}\right)$ for $H_{0}<\mu_{H}$ (degraded regime).

${ }^{23}$ Similarly, it is likely that were travelers to be patient (meaning that congestion at one instant can spill over to various departures), the mean congestion cost would be more regular and would always increase with headway variability. We could not confirm this point, however.
} 
reliability level. The relative values of the VoSR and the VoSR can help guide investments aiming to improve the quality of service towards either frequency or reliability improvements.

The analysis is subject to a certain number of caveats. First and foremost, in-vehicle travel time variability is not modeled. The extent to which modeling the variability of both headways and in-vehicle times would change our findings should be investigated by future research. The extension to congestion could be refined further and take into account the fact that travelers may want to wait for the next train in case of heavy congestion (in which case the phenomenon of "bunching" should also be represented). Last, the extent to which our results depend on the choice of $(\alpha, \beta, \gamma)$ scheduling preferences has also to be established.

\section{Acknowledgments}

We thank Transilien for making their database available, and Fabien Leurent and François Combes who contributed in stimulating this paper. We also wish to thank participants of the TSU seminar in Tokyo, in particular Mogens Fosgerau, for comments and for providing useful references.

\section{Appendix A. Mathematical appendix}

\section{A.1 Optimal head start}

For reminder, the optimal head start solves:

$$
\Phi_{w}\left(m^{*}\right)=\frac{\gamma}{\beta+\gamma}=\kappa
$$

We start by showing $m^{*} \geq m_{d e t}^{*}$. When $\sigma_{H}=0$, the p.d.f. of waiting times is: $\varphi_{W, \operatorname{det}}(x)=1 / \mu_{H}$ for $x \in\left[0, \mu_{H}\right]$. As $\frac{1-\Phi_{H}(x)}{\mu_{H}} \leq \frac{1}{\mu_{H}}$, we have $\varphi_{w}(x) \leq \varphi_{w, \operatorname{det}}(x)$ and thus $\Phi_{w}(x) \leq \Phi_{w, \operatorname{det}}(x) \forall x \in \mathrm{R}$. Considering that $\Phi_{w}\left(m^{*}\right)=\Phi_{w, \operatorname{det}}\left(m_{\text {det }}^{*}\right)=\kappa, m^{*} \geq m_{\text {det }}^{*}$ follows.

We then compute the derivatives of $m^{*}$ with respect to $\mu_{H}$ and $\sigma_{H}$. Rewrite $\Phi_{w}\left(m^{*}\right)$ as:

$$
\Phi_{w}\left(m^{*}\right)=\int_{0}^{m^{*}} \varphi_{w}(x) d x=\int_{0}^{m^{*}} \frac{1-\Phi_{H}(x)}{\mu_{H}} d x=\int_{0}^{m^{*}} \frac{1-\Phi_{h}\left(\frac{x-\mu_{H}}{\sigma_{H}}\right)}{\mu_{H}} d x
$$

(A.1) is therefore equivalent to:

$$
\int_{0}^{m^{*}} 1-\Phi_{h}\left(\frac{x-\mu_{H}}{\sigma_{H}}\right) d x=\kappa \mu_{H}
$$

Differentiating (A.2) with respect to $\mu_{H}$ yields:

$$
\frac{d m^{*}}{d \mu_{H}}\left[1-\Phi_{h}\left(\frac{m^{*}-\mu_{H}}{\sigma_{H}}\right)\right]+\left[\Phi_{h}\left(\frac{m^{*}-\mu_{H}}{\sigma_{H}}\right)-\Phi_{h}\left(-\frac{\mu_{H}}{\sigma_{H}}\right)\right]=\kappa
$$

The assumption of positive headways implying $\Phi_{h}\left(-\mu_{H} / \sigma_{H}\right)=\Phi_{H}(0)=0$, we have: 


$$
\frac{d m^{*}}{d \mu_{H}}=\frac{\kappa-\Phi_{H}\left(m^{*}\right)}{1-\Phi_{H}\left(m^{*}\right)}=\frac{\Phi_{w}\left(m^{*}\right)-\Phi_{H}\left(m^{*}\right)}{1-\Phi_{H}\left(m^{*}\right)}
$$

Since $\kappa<1, d m * / d \mu_{H}<1$ follows. The denominator being strictly positive, the sign of $d m * / d \mu_{H}$ is the same as for the numerator. The sign of $\Phi_{w}-\Phi_{H}$ depends on the shape of the headway distribution. While in most cases, including the deterministic and exponential cases, $\Phi_{w}(x) \geq \Phi_{H}(x)$, the opposite can occur for fat-tailed distributions (an example is available from the authors upon request).

Differentiating (A.2) with respect to $\sigma_{H}$ yields:

$$
\frac{d m^{*}}{d \sigma_{H}}\left[1-\Phi_{H}\left(m^{*}\right)\right]+\int_{0}^{m^{*}} \frac{x-\mu_{H}}{\sigma_{H}^{2}} \varphi_{h}\left(\frac{x-\mu_{H}}{\sigma_{H}}\right) d x=0
$$

Integrating by parts gives:

$$
\frac{d m^{*}}{d \sigma_{H}}=\frac{1}{\sigma_{H}}\left(m^{*}+\frac{\Phi_{H}\left(m^{*}\right)-\kappa}{1-\Phi_{H}\left(m^{*}\right)} \mu_{H}\right)
$$

Using $\beta>0$ and $m^{*} \geq m_{d e t}^{*}=\kappa \mu_{H}>0$, we have $\frac{d m^{*}}{d \sigma_{H}} \geq(1-\kappa) \frac{\mu_{H}}{\sigma_{H}} \frac{\Phi_{H}\left(m^{*}\right)}{1-\Phi_{H}\left(m^{*}\right)}>0$.

\section{A.2 VoSH and VoSR}

Using (5) and (8), we rewrite (18) as:

$$
c^{*}=\alpha_{v} T_{v}+\left(\alpha_{w}+\gamma\right) \mu_{w}-(\beta+\gamma) \int_{0}^{m^{*}} \frac{x}{\mu_{H}}\left(1-\Phi_{h}\left(\frac{x-\mu_{H}}{\sigma_{H}}\right)\right) d x
$$

We derive the VoSH:

$$
\begin{gathered}
\operatorname{VoSH}=\frac{\alpha_{w}+\gamma}{2}\left(1-\frac{\sigma_{H}^{2}}{\mu_{H}^{2}}\right)-(\beta+\gamma) \frac{d m^{*}}{d \mu_{H}} \frac{m^{*}}{\mu_{H}}\left(1-\Phi_{H}\left(m^{*}\right)\right)+\frac{\beta+\gamma}{\mu_{H}} \int_{0}^{m^{*}} x\left(\varphi_{w}(x)-\varphi_{H}(x)\right) d x \\
\Leftrightarrow \mathrm{VoSH}=\frac{\alpha_{w}+\gamma}{2}\left(1-\frac{\sigma_{H}^{2}}{\mu_{H}^{2}}\right)-\left(\gamma-(\beta+\gamma) \Phi_{H}\left(m^{*}\right)\right) \frac{m^{*}}{\mu_{H}}+\frac{\beta+\gamma}{\mu_{H}} m^{*}\left[\kappa-\Phi_{H}\left(m^{*}\right)\right] \\
+\frac{\beta+\gamma}{\mu_{H}} \int_{0}^{m^{*}}\left(\Phi_{H}(x)-\Phi_{w}(x)\right) d x
\end{gathered}
$$

This simplifies to:

$$
\operatorname{VoSH}=\frac{\alpha_{w}+\gamma}{2}\left(1-\frac{\sigma_{H}^{2}}{\mu_{H}^{2}}\right)+\frac{\beta+\gamma}{\mu_{H}} \int_{0}^{m^{*}}\left(\Phi_{H}(x)-\Phi_{w}(x)\right) d x
$$

We now derive the VoSR:

$$
\operatorname{VoSR}=\left(\alpha_{w}+\gamma\right) \frac{\sigma_{H}}{\mu_{H}}-(\beta+\gamma) \frac{d m^{*}}{d \sigma_{H}} \frac{m^{*}}{\mu_{H}}\left(1-\Phi_{H}\left(m^{*}\right)\right)-\frac{\beta+\gamma}{\mu_{H}} \int_{0}^{m^{*}} x \frac{x-\mu_{H}}{\sigma_{H}} \varphi_{H}(x) d x
$$




$$
\begin{aligned}
\Leftrightarrow \operatorname{VoSR}= & \left(\alpha_{w}+\gamma\right) \frac{\sigma_{H}}{\mu_{H}}-\frac{\beta+\gamma}{\sigma_{H}} \frac{m^{*}}{\mu_{H}}\left[m^{*}\left(1-\Phi_{H}\left(m^{*}\right)\right)+\mu_{H}\left(\Phi_{H}\left(m^{*}\right)-\kappa\right)\right] \\
& -\frac{\beta+\gamma}{\mu_{H}} \frac{m^{*}\left(m^{*}-\mu_{H}\right)}{\sigma_{H}} \Phi_{H}\left(m^{*}\right)+\frac{\beta+\gamma}{\mu_{H} \sigma_{H}} \int_{0}^{m^{*}}\left(2 x-\mu_{H}\right)\left(1-\mu_{H} \varphi_{w}(x)\right) d x
\end{aligned}
$$

Integrating by parts the last term and simplifying yields:

$$
\operatorname{VoSR}=\left(\alpha_{w}+\gamma\right) \frac{\sigma_{H}}{\mu_{H}}+\gamma \frac{\mu_{H}-m^{*}}{\sigma_{H}}-(\beta+\gamma) \frac{m^{*}}{\sigma_{H}}+2 \frac{\beta+\gamma}{\sigma_{H}} \int_{0}^{m^{*}} \Phi_{w}(x) d x
$$

\section{A.3 Exponential case}

In the exponential case, according to (11) we have:

$$
\begin{cases}\varphi_{w}(x)=\frac{1}{\mu_{H}} & \text { for } 0 \leq x \leq \mu_{H}-\sigma_{H} \\ \varphi_{w}(x)=\frac{1}{\mu_{H}} e^{-\left(\frac{x+\sigma_{H}-\mu_{H}}{\sigma_{H}}\right)} & \text { for } x \geq \mu_{H}-\sigma_{H}\end{cases}
$$

Computation gives:

$$
\begin{cases}\Phi_{w}(x)=\frac{x}{\mu_{H}} & \text { for } 0 \leq x \leq \mu_{H}-\sigma_{H} \\ \Phi_{w}(x)=1-\frac{\sigma_{H}}{\mu_{H}} e^{-\left(\frac{x+\sigma_{H}-\mu_{H}}{\sigma_{H}}\right)} & \text { for } x \geq \mu_{H}-\sigma_{H}\end{cases}
$$

We seek to solve $\Phi_{w}\left(m^{*}\right)=\kappa$. Two regimes naturally emerge depending on whether $m^{*}$ is greater or lower than $\mu_{H}-\sigma_{H}$.

Assume $m^{*} \leq \mu_{H}-\sigma_{H}$. (A.8) gives $m^{*}=\kappa \mu_{H}$. We rewrite the condition $m^{*} \leq \mu_{H}-\sigma_{H}$ as $\kappa \mu_{H} \leq \mu_{H}-\sigma_{H}$, hence $\sigma_{H} \leq(1-\kappa) \mu_{H}$. The minimum expected trip cost is:

$$
c^{*}=\alpha_{v} T_{v}+\left(\alpha_{w}+\gamma\right) \mu_{w}-(\beta+\gamma) \int_{0}^{m^{*}} x \varphi_{w}(x) d x=\alpha_{v} T_{v}+\left(\alpha_{w}+\gamma\right) \mu_{w}-(\beta+\gamma) \frac{m^{*}}{2 \mu_{H}}
$$

Using $m^{*}=\kappa \mu_{H}$ and $\mu_{w}=\left(\mu_{H}^{2}+\sigma_{H}^{2}\right) / 2 \mu_{H}$ yields:

$$
C^{*}=\alpha_{v} T_{v}+\left(\alpha_{w}+\gamma\right) \mu_{w}-\frac{\gamma^{2}}{\beta+\gamma} \frac{\mu_{H}}{2}=C_{d e t}+\left(\alpha_{w}+\gamma\right) \frac{\sigma_{H}^{2}}{2 \mu_{H}}
$$

It is now easy to derive the values of service headway and reliability:

$$
\left\{\begin{array}{l}
\operatorname{VoSH}=\frac{\partial C^{*}}{\partial \mu_{H}}=\frac{\alpha_{w}+\gamma}{2}\left(1-\frac{\sigma_{H}^{2}}{\mu_{H}^{2}}\right)-\frac{\gamma^{2}}{\beta+\gamma} \frac{1}{2}=\left(1-\frac{\sigma_{H}^{2}}{\mu_{H}^{2}}\right) \frac{\alpha_{w}}{2}+\left(\frac{\beta}{\beta+\gamma}-\frac{\sigma_{H}^{2}}{\mu_{H}^{2}}\right) \frac{\gamma}{2} \\
\operatorname{VoSR}=\frac{\partial C^{*}}{\partial \sigma_{H}}=\left(\alpha_{w}+\gamma\right) \frac{\sigma_{H}}{\mu_{H}}
\end{array}\right.
$$


The second mode corresponds to the case $m^{*} \geq \mu_{H}-\sigma_{H}$, which is equivalent to $\sigma_{H} \geq(1-\kappa) \mu_{H}$. Again, using (A.8) gives:

$$
1-\frac{\sigma_{H}}{\mu_{H}} e^{-\left(\frac{m^{*}+\sigma_{H}-\mu_{H}}{\sigma_{H}}\right)}=\frac{\gamma}{\beta+\gamma} \Leftrightarrow \frac{\beta}{\beta+\gamma} \frac{\mu_{H}}{\sigma_{H}}=e^{-\left(\frac{m^{*}+\sigma_{H}-\mu_{H}}{\sigma_{H}}\right)} \Leftrightarrow m^{*}=\mu_{H}-\sigma_{H}+\sigma_{H} \ln \left(\frac{\beta+\gamma}{\beta} \frac{\sigma_{H}}{\mu_{H}}\right)
$$

The minimum expected trip cost is given by:

$$
\begin{gathered}
C^{*}=\alpha_{v} T_{v}+\left(\alpha_{w}-\beta\right) \mu_{w}+(\beta+\gamma) \int_{m^{*}}^{+\infty} x \varphi_{w}(x) d x \\
\Leftrightarrow C^{*}=\alpha_{v} T_{v}+\left(\alpha_{w}-\beta\right) \mu_{w}+\beta\left(m^{*}+\sigma_{H}\right) \\
\Leftrightarrow C^{*}=\alpha_{v} T_{v}+\alpha_{w} \mu_{w}+\beta\left(\frac{\mu_{H}^{2}-\sigma_{H}^{2}}{2 \mu_{H}}+\sigma_{H} \ln \left(\frac{\beta+\gamma}{\beta} \frac{\sigma_{H}}{\mu_{H}}\right)\right)
\end{gathered}
$$

We can use this last formulation to derive the VoSH and VoSR:

$$
\left\{\begin{array}{l}
\operatorname{VoSH}=\left(1-\frac{\sigma_{H}^{2}}{\mu_{H}^{2}}\right) \frac{\alpha_{w}}{2}+\left(1-\frac{\sigma_{H}}{\mu_{H}}\right)^{2} \frac{\beta}{2} \\
\mathrm{VoSR}=\frac{\sigma_{H}}{\mu_{H}} \alpha_{w}+\left(1-\frac{\sigma_{H}}{\mu_{H}}+\ln \left(\frac{1}{1-\kappa} \frac{\sigma_{H}}{\mu_{H}}\right)\right) \beta
\end{array}\right.
$$

\section{References}

Abdel-Aty, M.A., Kitamura, R., Jovanis, P.P., 1995. Investigating effect of travel time variability on route choice using repeated-measurement stated preference data. Transportation Research Record 1493, 39-45.

Algers, S., Hansen, S., Tegner, G., 1975. Role of waiting time, comfort, and convenience in modal choice for work trip. Transportation Research Record 534, 38-51.

Bates, J., Polak, J., Jones, P., Cook, A., 2001. The valuation of reliability for personal travel. Transportation Research Part E 37(2-3), 191-229.

Batley, R., 2007. Marginal valuations of travel time and scheduling, and the reliability premium. Transportation Research Part E 43(4), 387-408.

Beesley, M.E., 1965. The value of time spent in travelling: some new evidence. Economica 32(126), 174.

Börjesson, M., Eliasson, J., Franklin, J.P., 2012. Valuations of travel time variability in scheduling versus mean-variance models. Transportation Research Part B 46(7), 855-873.

Bowman, L.A., Turnquist, M.A., 1981. Service frequency, schedule reliability and passenger wait times at transit stops. Transportation Research Part A 15(6), 465-471.

Carrion, C., Levinson, D., 2012. Value of travel time reliability: A review of current evidence. Transportation Research Part A 46(4), 720-741.

Chen, S., Liu, D., 2011. Assessing the influence of public transit headway irregularity on passenger's distribution. Presented at the Advanced Forum on Transportation of China (AFTC 2011), 7th, pp. $130-135$.

Chorus, C.G., Molin, E.J.E., van Wee, B., 2006. Travel information as an instrument to change cardrivers' travel choices: a literature review. European Journal of Transport and Infrastructure Research 6(4), 335-364. 
Coulombel, N., de Palma, A., 2012. The value of reliability: an equilibrium approach. Procedia - Social and Behavioral Sciences 54, 946-955.

Coulombel, N., de Palma, A., 2013. The variability of travel time, congestion, and the cost of travel. Mathematical Population Studies, in press.

Fosgerau, M., 2009. The marginal social cost of headway for a scheduled service. Transportation Research Part B 43(8-9), 813-820.

Fosgerau, M., Engelson, L., 2011. The value of travel time variance. Transportation Research Part B $45(1), 1-8$.

Fosgerau, M., Karlström, A., 2010. The value of reliability. Transportation Research Part B 44(1), 3849.

Furth, P., Muller, T., 2006. Service reliability and hidden waiting time: insights from automatic vehicle location data. Transportation Research Record 1955, 79-87.

Gaver, D.P., 1968. Headstart Strategies for Combating Congestion. Transportation Science 2(2), 172181.

Kleinrock, L., 1975. Queueing Systems. Volume 1: Theory, Volume 1. ed. Wiley-Interscience.

Knight, T.E., 1974. An approach to the evaluation of changes in travel unreliability: A "Safety margin" hypothesis. Transportation 3(4), 393-408.

Li, Z., Hensher, D.A., Rose, J.M., 2010. Willingness to pay for travel time reliability in passenger transport: A review and some new empirical evidence. Transportation Research Part E 46(3), 384-403.

Liu, H.X., Recker, W., Chen, A., 2004. Uncovering the contribution of travel time reliability to dynamic route choice using real-time loop data. Transportation Research Part A 38(6), 435-453.

Noland, R.B., Small, K.A., 1995. Travel-time uncertainty, departure time choice, and the cost of morning commutes. Transportation Research Record 1493, 150-158.

Noland, R.B., Small, K.A., Koskenoja, P.M., Chu, X., 1998. Simulating travel reliability. Regional Science and Urban Economics 28(5), 535-564.

Osuna, E.E., Newell, G.F., 1972. Control Strategies for an Idealized Public Transportation System. Transportation Science 6(1), 52-72.

Polak, J., 1987. Travel time variability and departure time choice. Transport Studies Group Discussion Paper $n^{\circ} 15$, Polytechnic of Central London.

Siu, B.W.Y., Lo, H.K., 2009. Equilibrium Trip Scheduling in Congested Traffic under Uncertainty, in: Lam, W.H.K., Wong, S.C., Lo, H.K. (Eds.), Transportation and Traffic Theory 2009: Golden Jubilee. Springer US, pp. 147-167.

Small, K.A., 1982. The scheduling of consumer activities: work trips. American Economic Review 72(3), 467-79.

STRATEC, RAND Europe, 2004. Enquêtes qualitatives et quantitatives visant à évaluer les projets de fiabilisation des radiales ferrées en Ile-de-France, Phase 1.2, Recherche bibliographique (Technical Report for the Syndicat des Transports d'Ile de France). France.

Tseng, Y.-Y., Verhoef, E.T., 2008. Value of time by time of day: A stated-preference study. Transportation Research Part B 42(7-8), 607-618.

Vickrey, W.S., 1969. Congestion Theory and Transport Investment. American Economic Review 59(2), 251-60.

Wardman, M., 2004. Public transport values of time. Transport Policy 11(4), 363-377. 